\title{
A Finite Element-Analytical Method for Electromagnetic Field Analysis of Electric Machines With Free Rotation
}

\author{
Yuejin Zhang ${ }^{1}$, K. T. Chau ${ }^{2}$, J. Z. Jiang ${ }^{1}$, Dong Zhang ${ }^{1}$, and Chunhua Liu ${ }^{2}$ \\ ${ }^{1}$ Department of Automation, Shanghai University, Shanghai 200072, China \\ ${ }^{2}$ Department of Electrical and Electronic Engineering, University of Hong Kong, Hong Kong, China
}

\begin{abstract}
A hybrid approach, namely the finite element-analytical method (FEAM), is newly proposed and implemented for electromagnetic field analysis of electric machines with free rotation. The key is to derive a new analytical expression of the airgap field which can naturally couple with the finite element fields of both the stator and rotor. The proposed FEAM is exemplified by using two practical machines. The accuracy and validity are verified by comparing with both the finite element method and experimental results.
\end{abstract}

Index Terms-Analytical solution, electric machines, electromagnetics, finite element method, hybrid approach.

\section{INTRODUCTION}

W ITH ever increasing demand of machine performance, the corresponding analysis approaches are continually fueled by computational electromagnetics. The analytical approach takes the advantages of fast computation and closed solution, but is ill-suited to practical machines with complicated or new structure [1]. The numerical approach can readily be applied to various machine structures, but generally suffers from lengthy computational time. The finite element method (FEM) is the most popular numerical approach. Since the knowledge of parameter variations with respect to rotor positions is crucial for the machine designer, the FEM is further extended by incorporating time stepping [2]. However, as the machine rotates, the finite elements in the airgap are inevitably distorted, leading to deteriorate the accuracy. In order to solve the aforementioned problem, the concept of hybrid approaches [3], [4] was proposed which combines both the analytical solution and the FEM. In [3], the Neumann boundary condition was used to couple between the analytical solution in the airgap region and the FEM equations in the stator and rotor regions. However, the calculation of Neumann condition involves additional computation. In [4], the Neumann condition was assumed to be zero. But, it is inappropriate. Also, these two hybrid methods were exemplified by using idealized or slotless machines, which are far from enough to testify their accuracy and validity.

In this paper, a hybrid approach, namely the finite elementanalytical method (FEAM), is newly proposed and implemented for electromagnetic field analysis of electric machines with free rotation. A new analytical solution of the airgap region will be derived, which can naturally couple with the FEM equations of both the stator and rotor regions.

Digital Object Identifier 10.1109/TMAG.2006.879441

\section{PROPOSED FEAM}

For a two-dimensional problem in electric machines, the magnetic field can be described as

$$
\nabla \times\left(\nu \nabla \times A_{z}\right)=J_{z}
$$

where $\nu$ is the magnetic reluctivity, $A_{z}$ is the magnetic vector potential, and $J_{z}$ is the current density. The variational formulation of (1) is by minimizing the energy functional $W$ :

$$
\left\{\begin{aligned}
W= & \iint_{\Omega}\left\{\left[\frac{\partial}{\partial x}\left(\nu \frac{\partial A_{z}}{\partial x}\right)+\frac{\partial}{\partial y}\left(\nu \frac{\partial A_{z}}{\partial y}\right)\right]-J_{z} A_{z}\right\} \\
& \cdot d x d y+\int_{s_{2}} H_{t} A_{z} d s \\
\mathrm{~S}_{1}: & A_{z}=A_{z 0}
\end{aligned}\right.
$$

where $\Omega$ is the solution domain, $S_{1}$ and $S_{2}$ are the Dirichlet and Neumann boundaries, respectively, and $H_{t}$ is the tangential component of magnetic field intensity on the Neumann boundary. In this paper, both the stator outside radius and rotor inside radius belong to $\mathrm{S}_{1}$, and $A_{z 0}$ is the boundary value of $A_{z}$ on $\mathrm{S}_{1}$ which is set to zero. By applying the standard FEM to (2), the system equation in matrix form can be obtained as

$$
\mathbf{S A}=\mathbf{F}
$$

where $\mathbf{S}$ is the stiffness matrix, $\mathbf{A}$ and $\mathbf{F}$ are the vectors of magnetic vector potentials and forcing functions, respectively.

In order to incorporate the analytical solution into the FEM, $W$ is divided into three components:

$$
W=W_{s}+W_{r}+W_{a}
$$

where $W_{s}$ and $W_{r}$ are the energy functionals of the stator and rotor regions, respectively, which will be handled by using the FEM, and $W_{a}$ is the energy functional of the airgap region which will be handled by analytical solution. It should be noted that the slots in the stator and rotor are incorporated by the stator and rotor regions, respectively; hence the slotting effects are inherently handled by the FEM. To couple the analytical solution of the airgap region with the FEM equation of both the stator and rotor regions, both the stator-airgap and rotor-airgap boundaries need to satisfy the Neumann condition. Since these boundaries 
can be selected in such a way that there are no current sources involved, $A_{z}$ must maintain continuity across the boundaries. Therefore, instead of directly calculating $H_{t}$ to ensure its continuity, the natural continuity of $A_{z}$ is simply adopted as the coupling scheme.

Because of no current source in the airgap region, the magnetic field can be described by the Laplace equation:

$$
\frac{\partial^{2} A_{z}}{\partial r^{2}}+\frac{1}{r} \frac{\partial A_{z}}{\partial r}+\frac{1}{r^{2}} \frac{\partial^{2} A_{z}}{\partial \theta^{2}}=0
$$

where $R_{r}<r<R_{s}, R_{r}$ is the rotor-airgap boundary radius, and $R_{s}$ is the stator-airgap boundary radius. So, the general solution of (5) is given by

$$
\begin{aligned}
A_{Z}(r, \theta) & =\sum_{n=1}^{\infty}\left[\left(A_{n} r^{p n}+B_{n} r^{-p n}\right) \cos (n p \theta)\right. \\
& \left.+\left(C_{n} r^{p n}+D_{n} r^{-p n}\right) \sin (n p \theta)\right]+A_{0} \ln r+B_{0}
\end{aligned}
$$

where $A_{n}, B_{n}, C_{n}, D_{n}, A_{0}$ and $B_{0}$ are constants, and $p$ is the number of periodicity which is greater or equal to unity. If the machine adopts integral-slot windings, $p$ will be the number of pole pairs. If adopting fractional-slot windings, $p$ will be the number of equivalent pole pairs according to the periodicity. From (6), the two airgap flux density components $B_{r}=$ $(1 / r) \partial A_{z} / \partial \theta, B_{\theta}=-\partial A_{z} / \partial r$ can readily be calculated. The general expression of $W_{a}$ in (4) is given by

$$
W_{a}=\int_{\Omega} \int \frac{1}{2} \nu_{0} B^{2} d \Omega=\int_{0}^{2 \pi / p} \frac{1}{2} \nu_{0} d \theta \int_{R_{r}}^{R_{s}} B^{2} r d r .
$$

Hence, by substituting $B_{r}$ and $B_{\theta}$ into (7), it yields

$$
\begin{aligned}
& W_{a}=\frac{\nu_{0} \pi}{2} \sum_{n=1}^{\infty}(n p)\left[\left(A_{n}^{2}+C_{n}^{2}\right)\left(R_{s}^{2 p n}-R_{r}^{2 p n}\right)\right. \\
& \left.-\left(B_{n}^{2}+D_{n}^{2}\right)\left(R_{s}^{-2 p n}-R_{r}^{-2 p n}\right)\right]+\pi \nu_{0} A_{0}^{2} \ln \left(\frac{R_{s}}{R_{r}}\right) .
\end{aligned}
$$

The values of $A_{z}$ at both the stator-airgap and rotor-airgap boundaries can be expressed by Fourier series in terms of the coefficients $a_{0}, a_{n}, b_{n}, c_{0}, c_{n}$, and $d_{n}$. Comparing them with the coefficients in (6), the constants $A_{n}, B_{n}, C_{n}, D_{n}, A_{0}$, and $B_{0}$ can readily be deduced. Hence, by substituting these constants into (8) and defining $q=R_{s} / R_{r}, W_{a}$ can finally be obtained as

$$
\begin{gathered}
W_{a}=\frac{\pi \nu_{0}}{2} \sum_{n=1}^{\infty} \frac{n}{q^{2 p n}-1}\left[\left(a_{n}^{2}+b_{n}^{2}+c_{n}^{2}+d_{n}^{2}\right)\left(q^{2 p n}+1\right)\right. \\
\left.-4\left(a_{n} c_{n}+b_{n} d_{n}\right) q^{\mathrm{pn}}\right]+\frac{\pi \nu_{0}}{4 \ln q}\left(a_{0}-c_{0}\right)^{2} .
\end{gathered}
$$

By minimizing $W_{a}$ with respect to $A_{s}$ and $A_{r}$ on the statorairgap and rotor-airgap boundaries, respectively, their contributions to the matrix $\mathbf{S}$ can be derived. In this way, the difference between the proposed FEAM and the standard FEM is the modification of $\mathbf{S}$ in (3). It can also be deduced that the contributions of $W_{a}$ to the matrix $\mathbf{S}$ are symmetric. Since the modified matrix retains the property of symmetry, the traditional computational approach developed for the FEM can be directly employed by the proposed FEAM.

It should be noted that the mesh grids within the stator and rotor regions keep unchanged when the rotor freely moves to

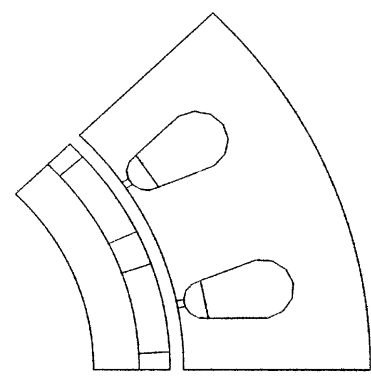

(a)

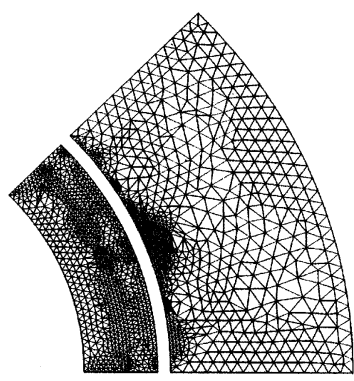

(b)
Fig. 1. Machine I. (a) Interested domain. (b) Meshes.

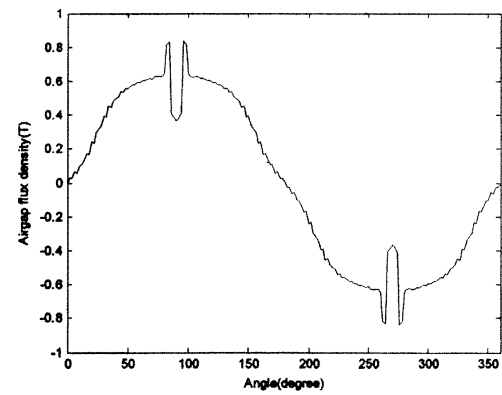

Fig. 2. Airgap flux density distribution of Machine I using FEAM.

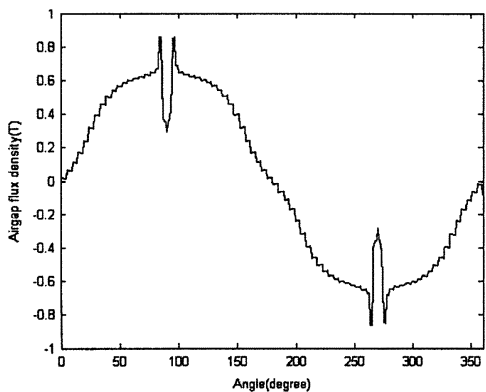

Fig. 3. Airgap flux density distribution of Machine I using FEM.

any position. The necessary work is to simply perform the coordinate transformation of rotor nodes for each rotation. This feature is very important to calculate those position-dependent parameters such as the back EMF, inductances, and torque, as well as the dynamic performances.

\section{VERIFICATION}

Firstly, numerical verification is performed. Rather than adopting an ideal or slotless machine, a practical permanent magnet brushless DC (PMBDC) machine with open-mouth slots, so-called the Machine I as shown in Fig. 1, is adopted because the variation of magnetic field nearby the slots is serious and its calculation is challenging. Fig. 2 shows the airgap flux density with the radius on the stator-airgap boundary resulted from using the FEAM in which there are 3389 elements (with no airgap element) and 1833 nodes, whereas Fig. 3 shows the counterpart resulted from using the standard FEM in which there are 3878 elements (with highly dense airgap elements) and 2008 nodes. It can be found that the agreement is very good, hence numerically verifying the accuracy of the proposed FEAM. It should be noted that the FEAM can save computational time due to the use of fewer number of nodes, but it 


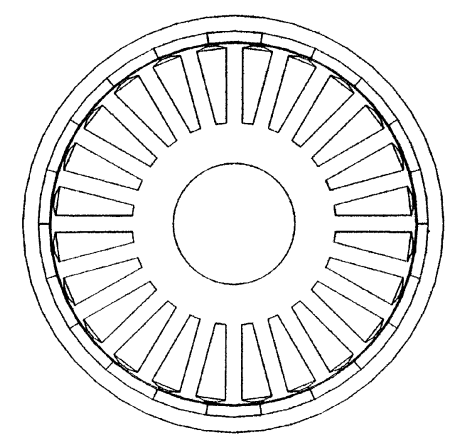

Fig. 4. Configuration of Machine II.

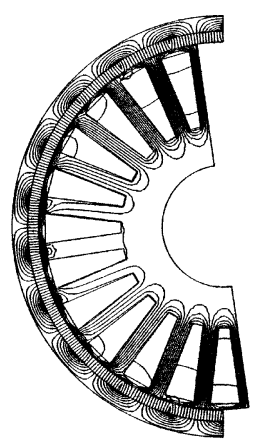

(a)

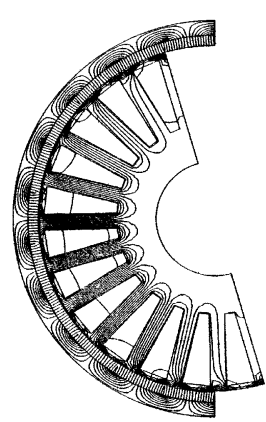

(b)
Fig. 5. Magnetic flux density distributions of Machine II at various rotor positions using FEAM. (a) $90^{\circ}$. (b) $180^{\circ}$.

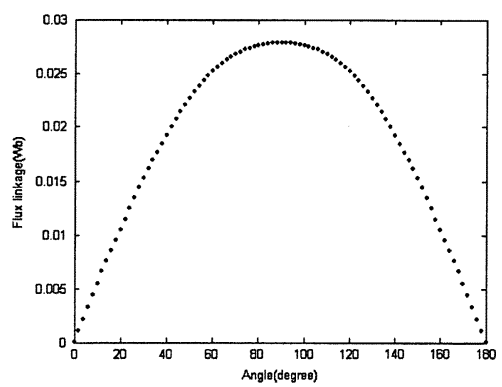

Fig. 6. Flux linkage versus rotor position of Machine II using FEAM.

needs additional time to calculate the analytical expressions and hence to modify the matrix $\mathbf{S}$. So, the overall computational time of the FEAM is similar to that of the FEM.

Secondly, experimental verification is performed. A practical outer-rotor PMBDC machine with 22 poles and 24 slots, so-called the Machine II as shown in Fig. 4, is prototyped for experimentation. To illustrate the unique feature of FEAM, the no-load EMF is calculated for each rotor step of $2^{\circ}$. Fig. 5 shows the magnetic flux density distributions of the Machine II at two typical rotor positions. The corresponding flux linkage and hence the no-load EMF waveforms are shown in Figs. 6 and 7, respectively. Under the same operating condition, the no-load EMF waveform of the prototype is measured as shown in Fig. 8. It can be found that the calculated and measured EMF waveforms have a very good agreement, hence confirming that the FEAM can allow for the machine with actual free rotation.

Therefore, it is verified that the FEAM takes the benefit of high accuracy when repetitive calculations under different rotor positions are required. Of course, the FEM still takes the benefit of high generality.

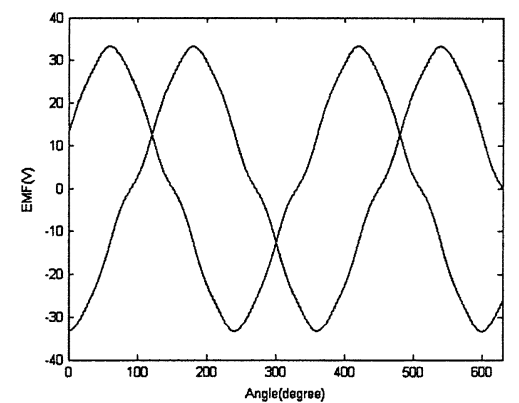

Fig. 7. Calculated no-load EMF waveforms of Machine II using FEAM.

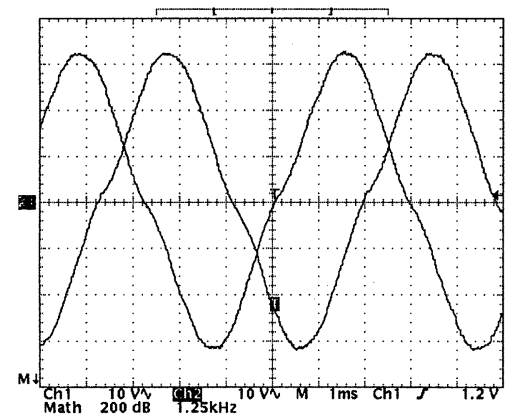

Fig. 8. Measured no-load EMF waveforms of Machine II (10 V/div, $1 \mathrm{~ms} / \mathrm{div})$.

\section{CONCLUSION}

A hybrid approach, namely the FEAM, has been presented for electromagnetic field analysis of electric machines with free rotation. Since there are no meshes in the airgap region, the problems of mesh distortion and highly dense airgap meshes are totally eliminated. The accuracy of the proposed FEAM has been numerically verified by comparing with the standard FEM in which highly dense airgap elements are required. Moreover, the validity of free rotation of the FEAM-analyzed machine has been experimentally verified by comparing with the measured no-load EMF waveforms.

\section{ACKNOWLEDGMENT}

This work was supported and funded in part by the Delta Science \& Technology Education Program, China, and the Hong Kong Research Grants Council under Grant HKU7111/05E, China.

\section{REFERENCES}

[1] M. Cheng, K. T. Chau, C. C. Chan, E. Zhou, and X. Huang, "Nonlinear varying-network magnetic circuit analysis for doubly salient permanent magnet motors," IEEE Trans. Magn., vol. 36, no. 1, pp. 339-348, Jan. 2000.

[2] Y. Wang, K. T. Chau, C. C. Chan, and J. Z. Jiang, "Transient analysis of a new outer-rotor permanent-magnet brushless dc drive using circuitfield-torque time-stepping finite element method," IEEE Trans. Magn., vol. 38, no. 2, pp. 1297-1300, Mar. 2002.

[3] K. Lee, M. J. DeBortoli, M. J. Lee, and S. J. Salon, "Coupling finite elements and analytical solution in the airgap of electric machines," IEEE Trans. Magn., vol. 27, no. 5, pp. 3955-3957, Sep. 1991.

[4] B. Chao, S. X. Chen, Z. J. Liu, and T. S. Low, "Electromagnetic field analysis in rotational electric machines using finite element-analytical hybrid method," IEEE Trans. Magn., vol. 30, no. 6, pp. 4314-4316, Nov. 1994.

Manuscript received November 30, 2005 (e-mail: ktchau@eee.hku.hk). 